Supporting Information for

\title{
Insensitivity of Sterically Defined Helical Chain Conformations to Solvent Quality in Dilute Solution
}

Beihang Yu, ${ }^{\dagger}$ Scott P. O. Danielsen, ${ }^{\dagger}$ Kai-Chieh Yang, ${ }^{\S}$ Rong-Ming Ho, ${ }^{\S}$ Lynn M. Walker, Rachel A. Segalman ${ }^{\dagger, \hat{*}, *}$

${ }^{\dagger}$ Department of Chemical Engineering, ${ }^{\star}$ Materials Department, University of California, Santa Barbara, Santa Barbara, California 93106, United States

${ }^{\S}$ Department of Chemical Engineering, National Tsing Hua University, Hsinchu 30010, Taiwan

"Department of Chemical Engineering, Center for Complex Fluids Engineering, Carnegie Mellon University, Pittsburgh, Pennsylvania 15213, United States

*Corresponding author: R.A.S. (segalman@ucsb.edu)

\section{Experimental Details}

\subsection{Materials}

Solvents and reagents were purchased from commercial suppliers and used without further purification, unless otherwise noted. HPLC-grade acetonitrile (ACN), tetrahydrofuran (THF), acetone (ACE), and $N, N$-dimethylformamide (DMF) were used for static light scattering measurements. Deuterated solvents $\left(\mathrm{ACN}-d_{3}, \mathrm{THF}-d_{8}, \mathrm{ACE}-d_{6}, \mathrm{DMF}-d_{7}\right)$ were purchased from Cambridge Isotope Laboratories, Inc. and used for small-angle neutron scattering measurements.

\subsection{Synthesis, purification and characterization of polypeptoids}

Polypeptoids were synthesized and purified using the same methods described elsewhere. ${ }^{1}$ Polypeptoid mass and purity were determined by UPLC-MS.

\subsection{Circular Dichroism (CD)}

CD measurements were performed on a J-1500 CD spectrometer. Polypeptoid solutions were prepared at a concentration of $0.1 \mathrm{mg} \mathrm{mL}^{-1}$, and CD spectra of the solutions were collected using quartz cells (Starna Cells) with a $1 \mathrm{~mm}$ path length. A scan speed of $200 \mathrm{~nm} \mathrm{~min}^{-1}$ was used, and 5 measurements were averaged for each sample. Vibrational circular dichroism (VCD) 
measurements were performed on a JASCO FVS-6000 VCD spectrometer. Polypeptoid solutions were prepared at a concentration of $26 \mathrm{mg} \mathrm{mL}^{-1}$, and VCD spectra of the solutions were collected using liquid cells with a $0.5 \mathrm{~mm}$ path length. A resolution of $4 \mathrm{~cm}^{-1} \mathrm{~min}^{-1}$ was used, and 4000 accumulations were averaged for each sample.

\subsection{Static light scattering (SLS)}

For each polypeptoid-solvent pair, 300-500 mg polypeptoids were dissolved in double filtered solvent and sonicated for $0.5 \mathrm{~h}$. The solution was then filtered twice (filtered with Teflon filter with $0.2 \mu \mathrm{m}$ pores) to remove dust particles and transferred to volumetric flask to make a $10 \mathrm{~mL}$ solution. The solution was then transferred to a $20 \mathrm{~mL}$ scintillation vial for SLS measurements. Volumetric flasks and scintillation vials used were rinsed with double filtered solvent and dried in an oven. SLS measurements were taken on a Brookhaven BI-200SM light scattering instrument with a HeNe diode laser at $532 \mathrm{~nm}$. The sample cell was equilibrated for $15 \mathrm{~min}$ in a Decalin bath with temperature set to $20^{\circ} \mathrm{C}$ before the measurements were taken. The solution was diluted to another 5 lower concentrations to get averaged scattering light intensity at angles of $90^{\circ}, 105^{\circ}, 120^{\circ}, 135^{\circ}$. The second virial coefficient, $A_{2}$, was extracted from the slope of $K c / R \sim c$. After SLS measurements, the solution was dried on a EZ-2 Genevac (SP Scientific) and materials were lyophilized from acetonitrile/water to determine the actual solution concentration for SLS. Refractive index increments were determined using a Waters e2695 GPC equipped with a 2414 refractive index detector. The flow cell in the RI detector was purged with corresponding solvents before injection of samples. For each polypeptoid-solvent pair, 3 concentrations were prepared and each injected with injection volumes of 10, 20, 30, $40 \mu \mathrm{L}$. Polystyrene (in THF and DMF) and poly(methyl methacrylate) (in ACN and ACE) solutions were measured in the same way to determine the calibration constant for the RI detector to give the correct refractive index increment $(d n / d c)$ value for each polypeptoid-solvent pair.

\subsection{Small-angle neutron scattering (SANS)}

SANS studies were conducted at the extended Q-range small-angle neutron scattering diffractometer line (EQ-SANS BL-6) at the Spallation Neutron Source (SNS) at Oak Ridge National Laboratory (ORNL). Samples were prepared at a concentration of $20 \mathrm{mg} \mathrm{mL}^{-1}$ in deuterated solvents (acetonitrile- $d_{3}$, tetrahydrofuran- $d_{8}$, aceton- $d_{6}, N, N$-dimethylformamide- $d_{7}$ ) to 
enhance contrast between the polypepoids and the solvents. Solutions were sonicated for $30 \mathrm{~min}$ and sit overnight to allow sufficient dissolution. Quartz banjo cells with a path length of $2 \mathrm{~mm}$ were used in a multiple position sample holder with temperature set to $20{ }^{\circ} \mathrm{C}$. 3 different configurations of sample-to-detector distance and neutron wavelength (4 m-2.5, 1.3 m-2.5 , and $1.3 \mathrm{~m}-1 \AA$ ) were used. Data reduction followed standard procedures implemented in MantidPlot. ${ }^{2}$ The measured intensity was corrected for detector sensitivity and the scattering contributions from the solvent and empty cells, and calibrated to an absolute scale using a Porasil B standard. ${ }^{3}$

\section{Polypeptoids Characterization}

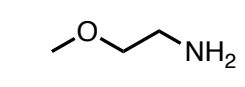

2-Methoxyethylamine

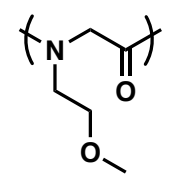

Nme
$N-(2-m e t h o x y e t h y l) g l y c i n e$

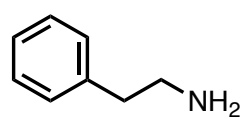

Phenylethylamine

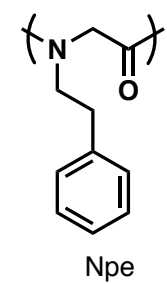

$N$-phenylethylglycine<smiles>C[C@H](N)c1ccccc1</smiles>

(R)-(+)- $\alpha$-Methylbenzylamine

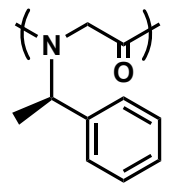

NRpe

$N-((R)-(+)-\alpha-$ methylbenzyl)glycine<smiles>C[C@H](N)c1ccccc1</smiles>

(S)-(-)- $\alpha$-Methylbenzylamine

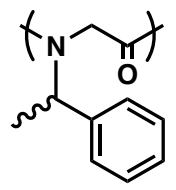

$\mathrm{Nmb}$

$N-((R / S)-( \pm)-\alpha-$ methylbenzyl)glycine

Figure S1. Chemical structures of amine submonomers used for polypeptoid synthesis and repeat glycine units of polypeptoids in this work.
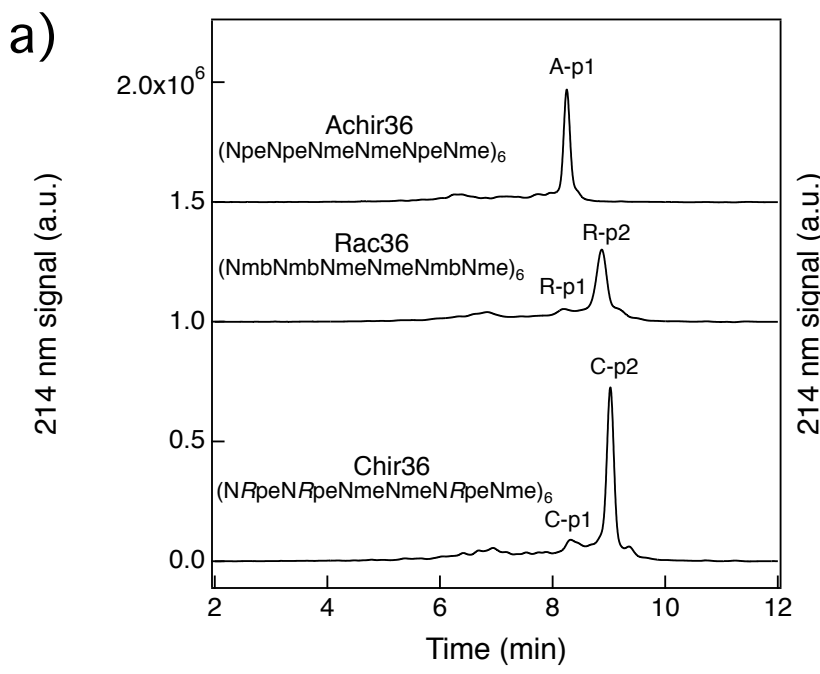

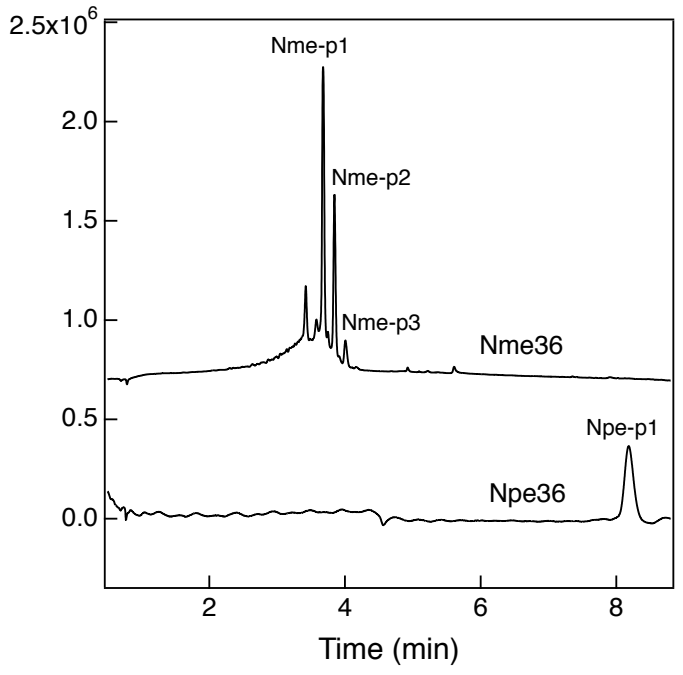


b)

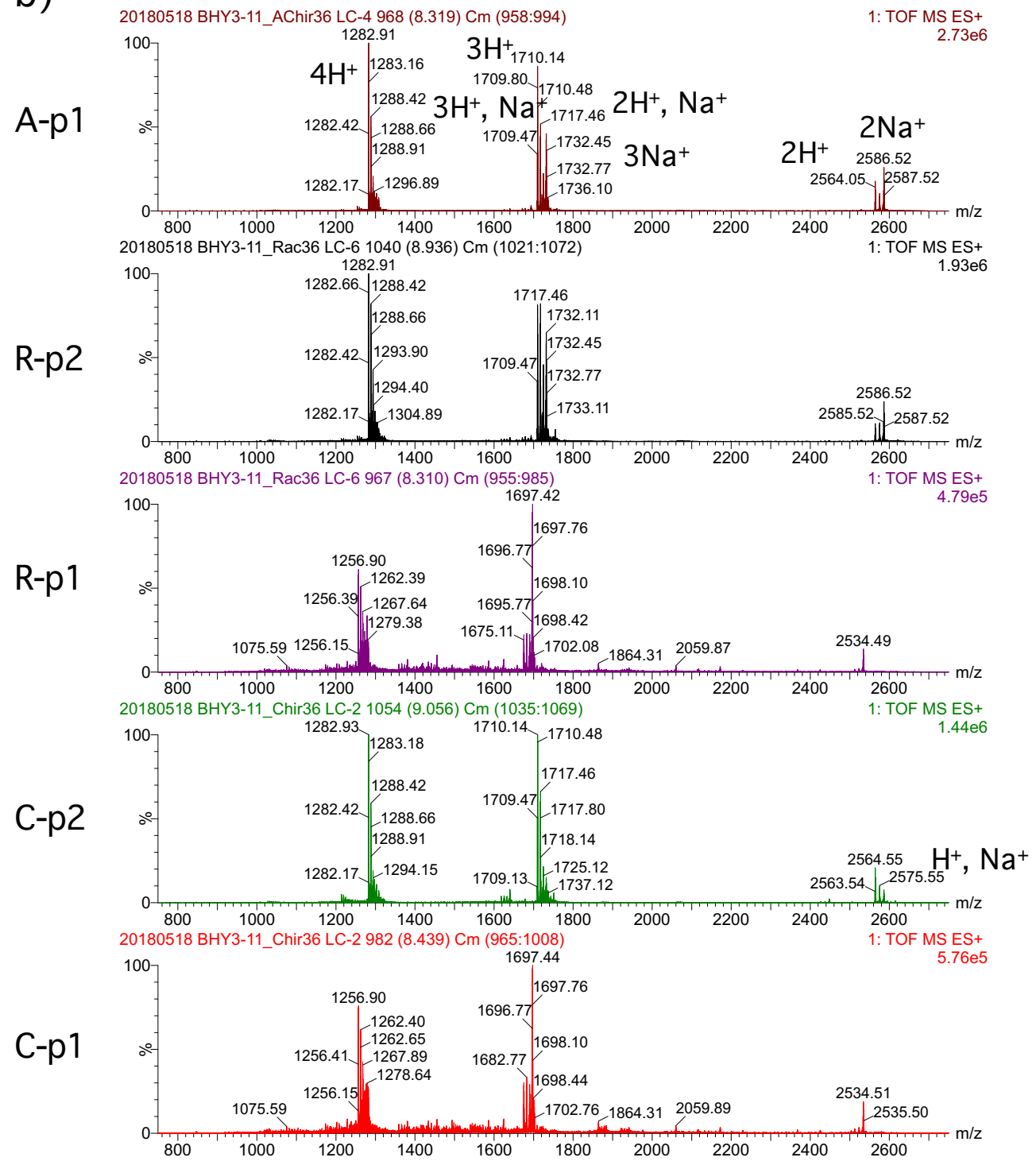




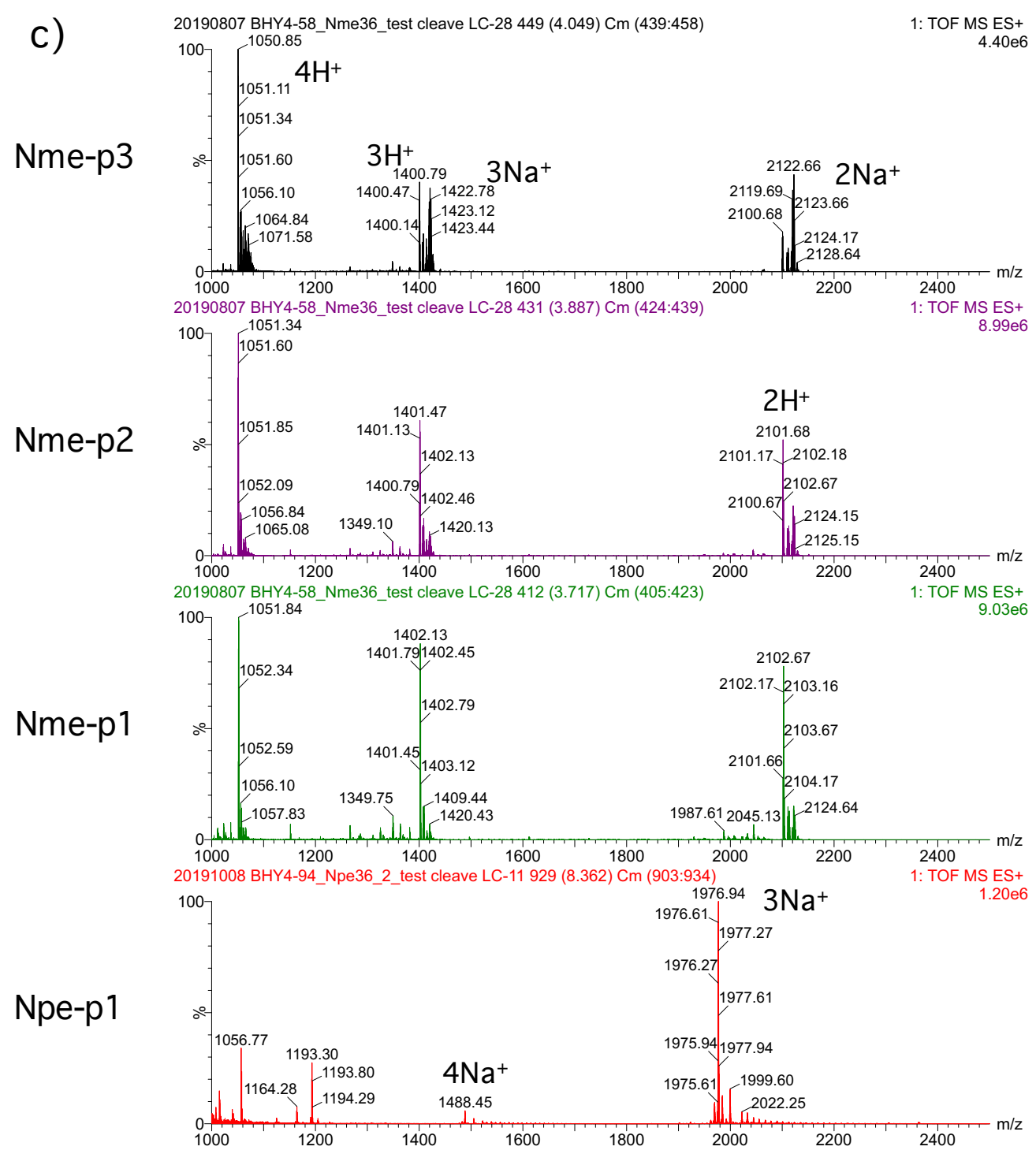

Figure S2. a) Polypeptoids were detected by UV absorption at $214 \mathrm{~nm}$ using UPLC-MS. b)

Doubly, triply, quadruply charged polypeptoid species were detected in the mass spectrometer.

Chir36, Rac36, and Achir36 have the same chemical formula and a molecular weight of $5128.2 \mathrm{~g}$ $\mathrm{mol}^{-1}$ (m/z: 5126.73 (100\%), 5127.74 (90.6 \%), ...). For Chir36 and Rac36, R-p1 and C-p1 before the main product peak are peaks of 36-mers with one methylbenzyl side chain (104 $\mathrm{g} \mathrm{mol}^{-}$ $\left.{ }^{1}\right)$ cleaved off (m/z: $\left.5022.67(100 \%)\right)$. c) Doubly, triply, quadruply charged polypeptoid species of Nme36 (MW: $4203.8 \mathrm{~g} \mathrm{~mol}^{-1}, \mathrm{~m} / \mathrm{z}: 4202.3$ (100\%), 4203.3 (97.9\%), ...) and Npe36 (MW: $\left.5862.4 \mathrm{~g} \mathrm{~mol}^{-1}, \mathrm{~m} / \mathrm{z}: 5861.1(100 \%), 5862.1(94.8 \%), \ldots\right)$. Nme-p1, p2, and $\mathrm{p} 3$ are peaks of the target product but with different charges, therefore eluded at different times. 


\section{Circular Dichroism (CD) and Vibrational Circular Dichroism (VCD)}
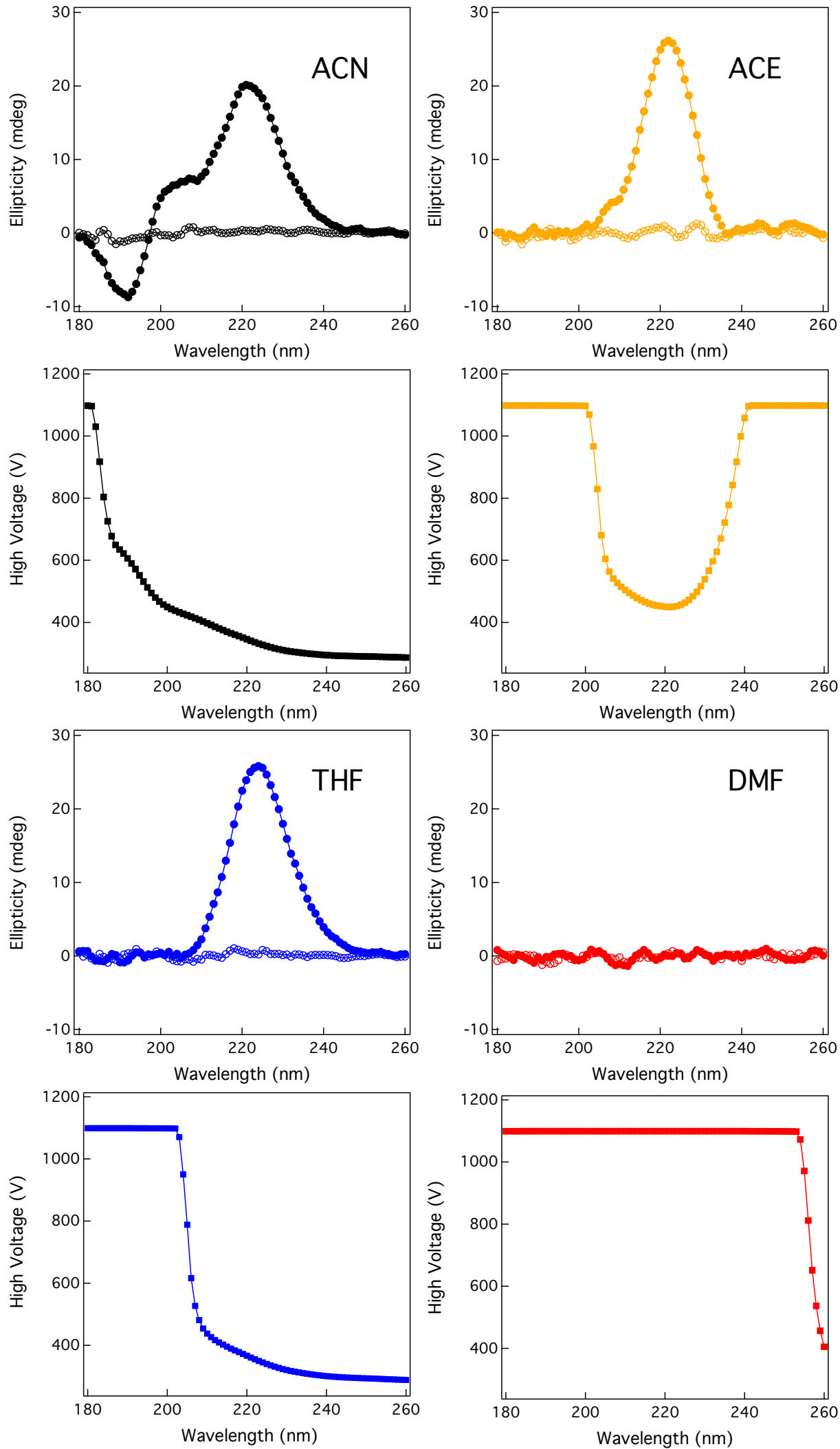
Figure S3. Circular dichroism (Chir36: filled symbols, Rac36: open symbols) and high voltage signals in different solvents. A high voltage signal higher than $650 \mathrm{~V}$ indicates few photons are reaching the detector, therefore, less reliable CD signal. In DMF, high voltage reached maximum $(1098 \mathrm{~V})$ at $\sim 255 \mathrm{~nm}$ and persisted to lower wavelengths. CD spectra in DMF in the wavelength range of interest are cut off by the solvent absorbance.

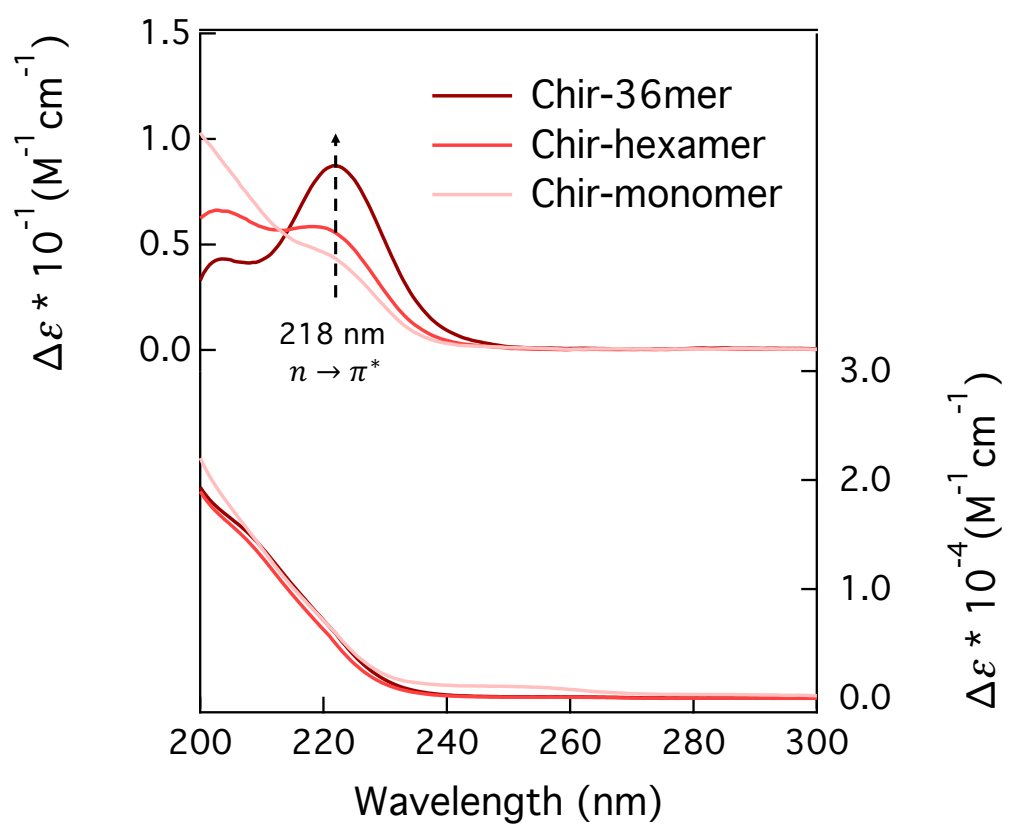

Figure S4. Circular dichroism (CD) spectra and corresponding UV absorption of Chiralmonomer, hexamer, and 36mer in dilute solution. The helical conformation stability of Chir36 can be recognized in CD with amplification of the $n \rightarrow \pi^{*}$ signal as the chain length increases. 


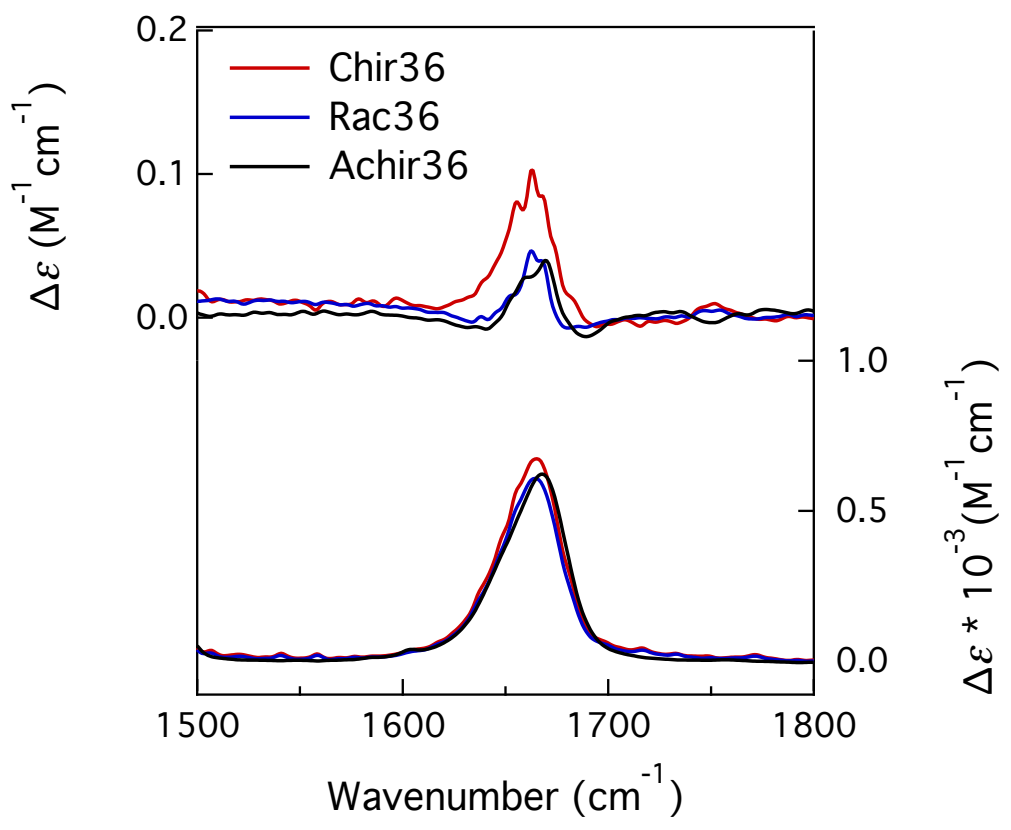

Figure S5. Vibrational circular dichroism (VCD) spectra and corresponding FT-IR absorption spectra of helical (Chir36) and coil (Rac36, Achir36) polypeptoids in dilute solution. The strong positive VCD signal at $1665 \mathrm{~cm}^{-1}(\mathrm{C}=\mathrm{O}$ stretching motions $)$ of Chir36 indicates a helical conformation adopted by the polypeptoid backbone. The slight positive VCD signals of Rac36 and Achir36 are attributed to the non-equal population between cis and trans backbone configurations, where neighboring glycine units both adopting a cis configuration will lead to a net VCD signal. 


\section{Static Light Scattering (SLS)}

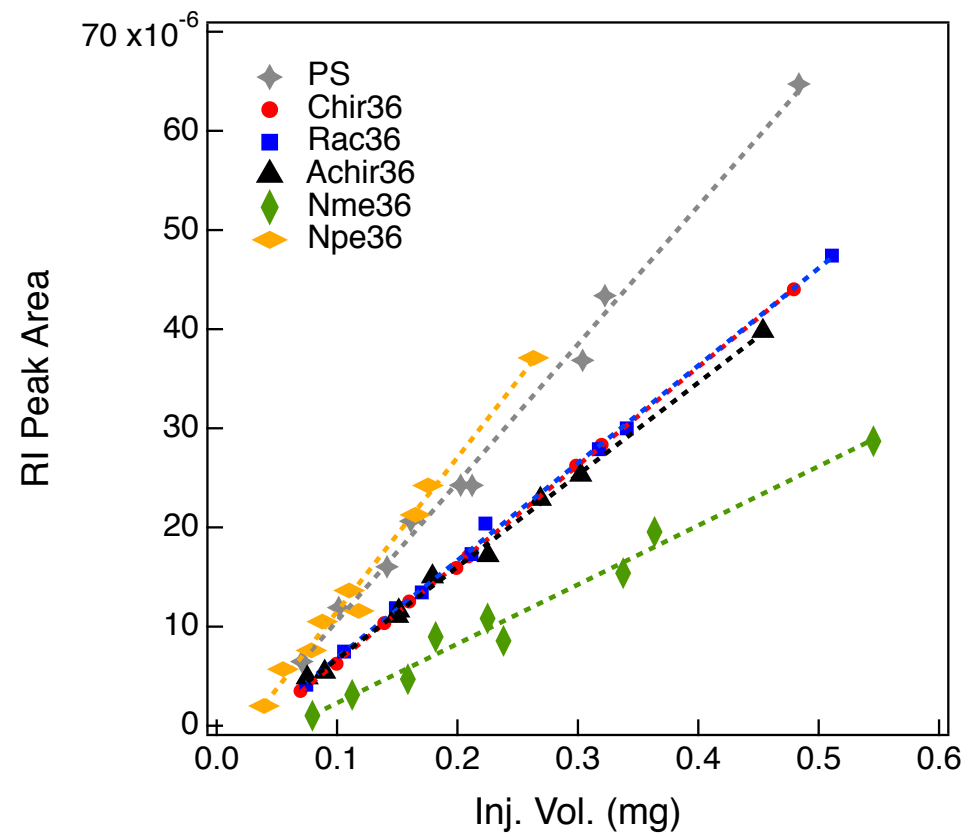

Figure S6. RI peak area vs. sample injection volume (mg) (data in DMF as an example). The slope of the line (linear fit with $95 \%$ confidence) is proportional to the refractive index increment $(d n / d c)$ and the absolute value of $d n / d c$ for each polypeptoid-solvent pair is given by calibrating against a polymer-solvent pair with known $d n / d c$ (here PS-DMF).

Table S1. Refractive index increment $(d n / d c)$ of each polymer-solvent pair.

\begin{tabular}{|c|c|c|c|c|c|}
\hline \multicolumn{2}{|c|}{ polymer-solvent pair } & $d n / d c\left(\mathrm{~mL} \mathrm{~g}^{-1}\right)$ & \multicolumn{2}{|c|}{ polymer-solvent pair } & $d n / d c\left(\mathrm{~mL} \mathrm{~g}^{-1}\right)$ \\
\hline \multirow{6}{*}{ THF } & PS & $0.185^{4}$ & \multirow{6}{*}{$\mathrm{ACE}$} & PMMA & $0.134^{5}$ \\
\hline & Chir36 & $0.129 \pm 0.006$ & & Chir36 & $0.206 \pm 0.007$ \\
\hline & Rac36 & $0.131 \pm 0.006$ & & $\operatorname{Rac} 36$ & $0.199 \pm 0.011$ \\
\hline & Achir36 & $0.131 \pm 0.006$ & & Achir36 & $0.204 \pm 0.010$ \\
\hline & Nme36 & $0.090 \pm 0.009$ & & Nme36 & $0.163 \pm 0.010$ \\
\hline & Npe36 & $0.141 \pm 0.013$ & & Npe36 & / \\
\hline \multirow{6}{*}{ DMF } & PS & $0.159^{4}$ & \multirow{6}{*}{$\mathrm{ACN}$} & PMMA & $0.137^{5}$ \\
\hline & Chir36 & $0.113 \pm 0.001$ & & Chir36 & $0.196 \pm 0.018$ \\
\hline & $\operatorname{Rac} 36$ & $0.112 \pm 0.004$ & & $\operatorname{Rac} 36$ & $0.193 \pm 0.020$ \\
\hline & Achir36 & $0.106 \pm 0.005$ & & Achir36 & $0.190 \pm 0.016$ \\
\hline & Nme36 & $0.068 \pm 0.009$ & & Nme36 & $0.160 \pm 0.016$ \\
\hline & Npe36 & $0.177 \pm 0.017$ & & Npe36 & / \\
\hline
\end{tabular}



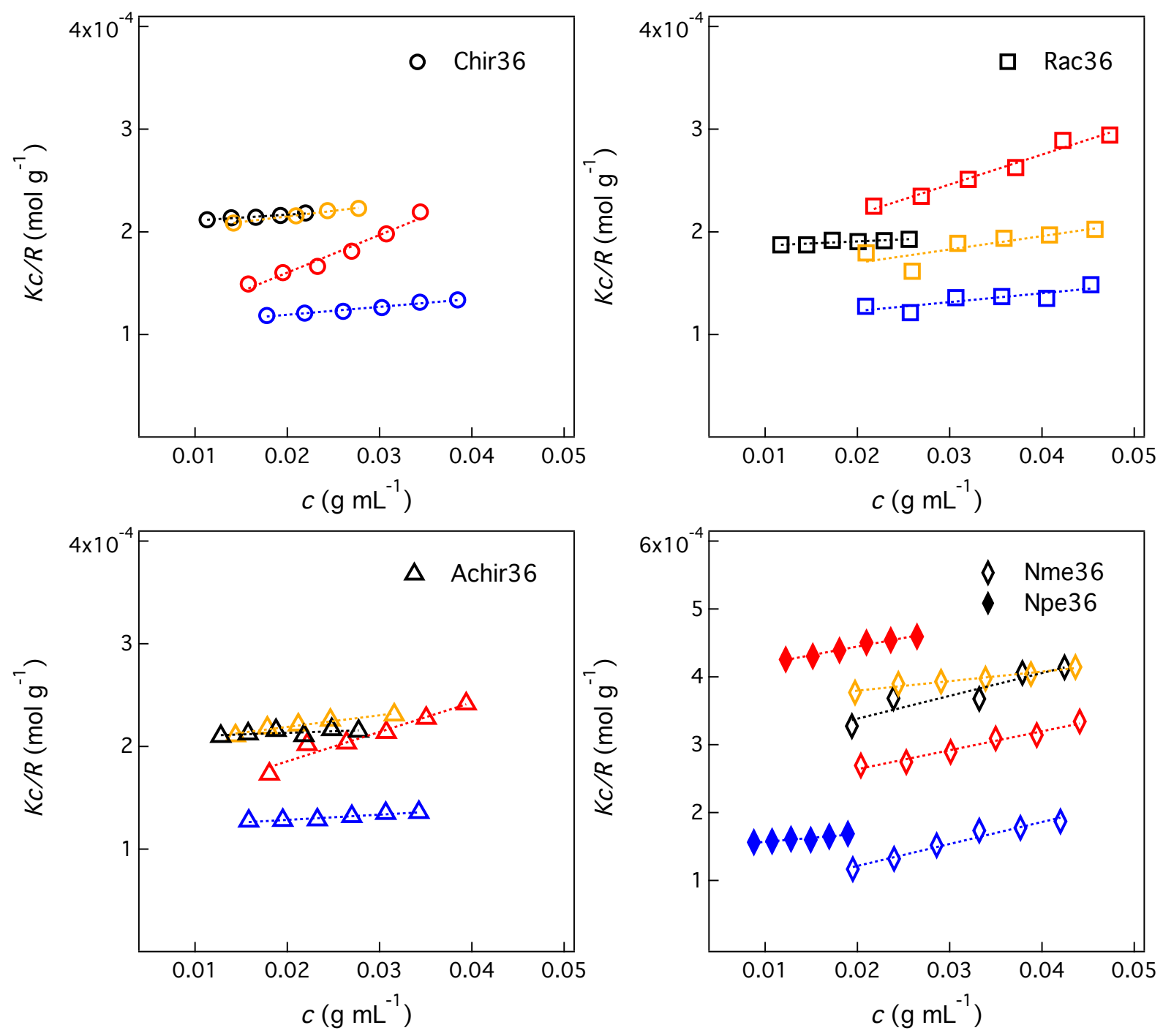

Figure S7. Summary of static light scattering (SLS) results of polypeptoids in different solvents.

The scattering intensity showed minimal angular dependence as these polypeptoids are relatively small. The secondary virial coefficient, $A_{2}$, is determined from the slope of $K c / R \sim c$, using averaged values of scattering intensity at angles of $90^{\circ}, 105^{\circ}, 120^{\circ}, 135^{\circ}$. 
Table S2. Second virial coefficient, $A_{2}$, of Achir36 and its corresponding homopolypeptoids (Nme36 and Npe36) in different solvents determined by static light scattering (SLS) at $20{ }^{\circ} \mathrm{C}$.

\begin{tabular}{ccccc}
\hline \multirow{2}{*}{ Solvent } & \multicolumn{3}{c}{ Second virial coefficient, $A_{2} * 10^{3}\left(\mathrm{~cm}^{3} \mathrm{~mol} \mathrm{~g}^{-2}\right)$} \\
& $A_{2, \text { Nme36 }}$ & $A_{2, \text { Npe36 }}$ & $A_{2, \text { Achir36_calc }}$ & $A_{2, \text { Achir36_measured }}$ \\
\hline ACN & $1.68 \pm 0.72$ & Insoluble & $/$ & $0.16 \pm 0.12$ \\
THF & $1.62 \pm 0.48$ & $0.58 \pm 0.21$ & $1.02 \pm 0.52$ & $0.26 \pm 0.06$ \\
ACE & $1.20 \pm 0.22$ & Insoluble & $/$ & $0.57 \pm 0.13$ \\
DMF & $1.40 \pm 0.48$ & $1.27 \pm 0.34$ & $1.32 \pm 0.59$ & $1.43 \pm 0.31$ \\
\hline
\end{tabular}

The homopolypeptoids with all chiral/racemic side chains (NRpe36/Nmb36) are not synthetically accessible. Here, solvent quality for corresponding homopolypeptoids of Achir36 (i.e., Nme36 and Npe36) were examined. The calculated second virial coefficient assumes a linear dependence of $A_{2}$ on monomer weight fractions.

\section{Small-Angle Neutron Scattering (SANS)}

\subsection{SANS of Chir36, Rac36 and Achir36 in dilute solutions}

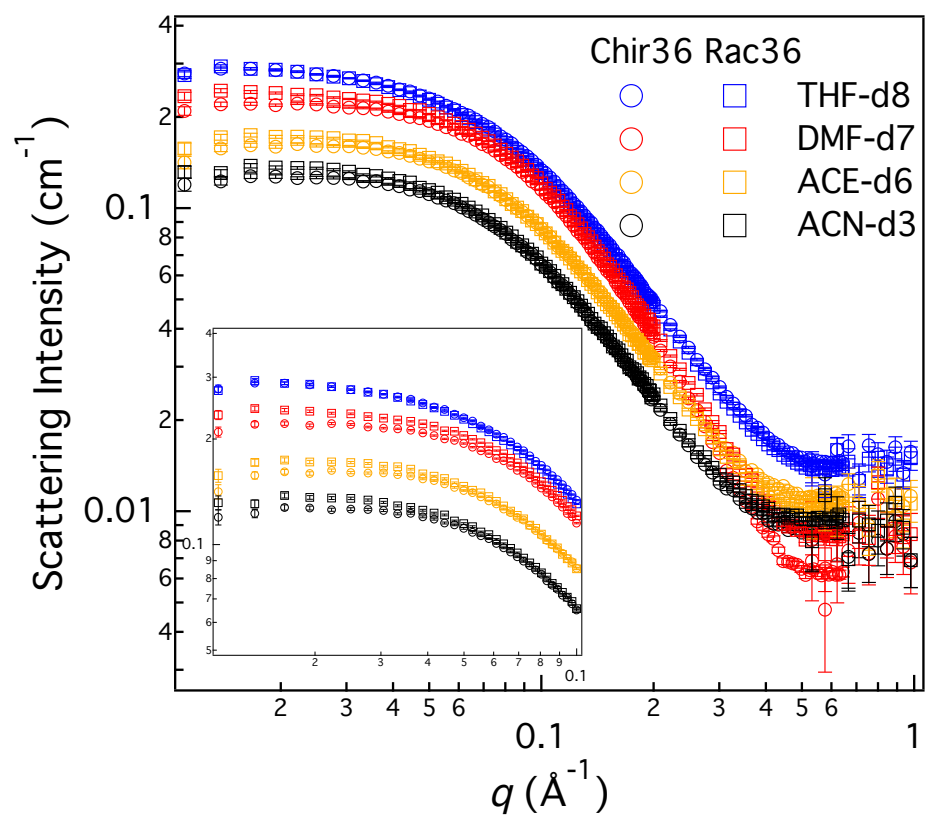

Figure S8. Scattering intensity comparison of Chir36 and Rac36 under the same dilute solution conditions. The inset shows the low $q$ region comparison in particular, where the scattering intensity of the helical polypeptoids (Chir36) is consistently lower than the chemically identical coil polypeptoids (Rac36), indicating the helical polypeptoids having a higher density. 

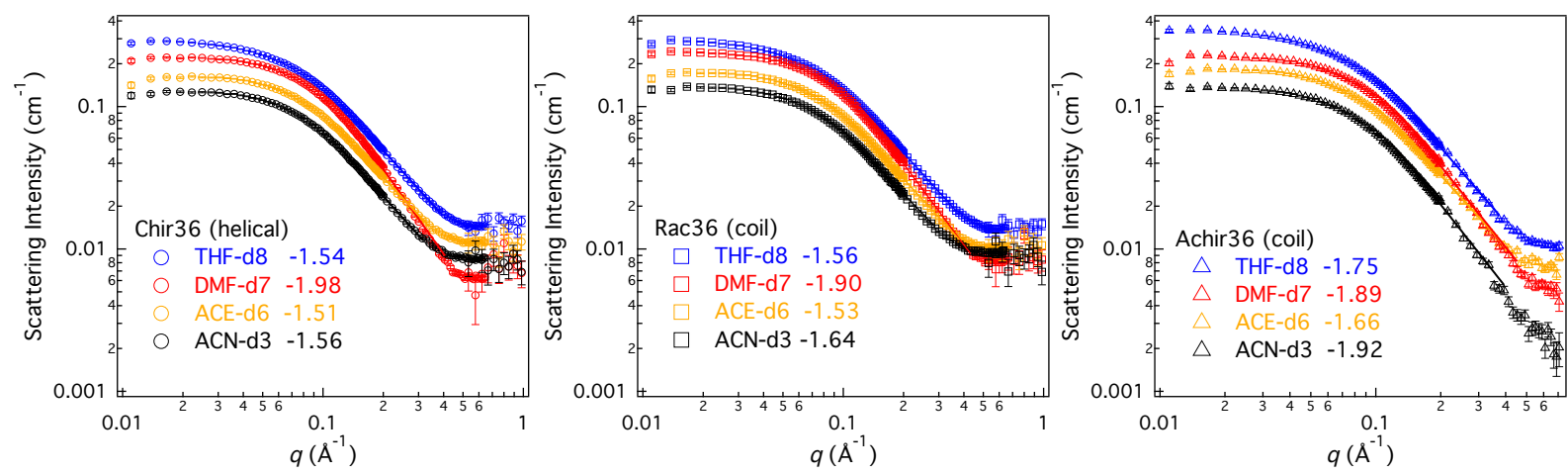

Figure S9. Porod scaling exponents of the three polypeptoids (Chir36, Rac36, Achir36) in dilute solutions (SANS data are solvent subtracted).

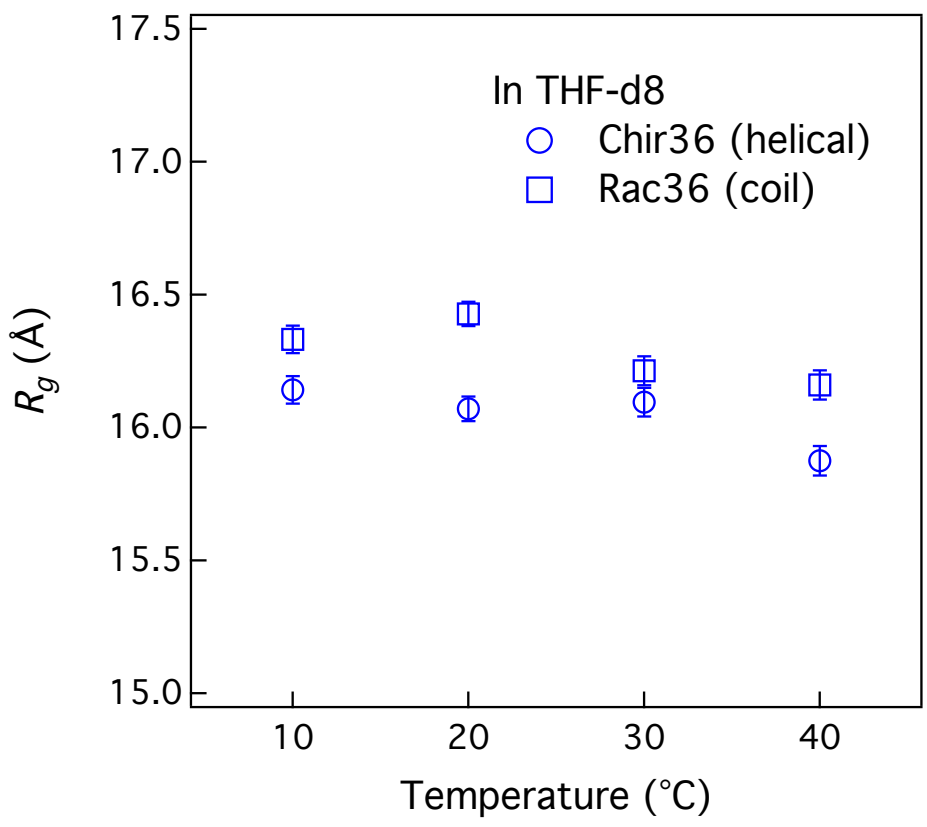

Figure $\mathrm{S} 10 . R_{g} \mathrm{~s}$ (determined from the slopes of Guinier plots) of Chir36 and Rac36 as a function of temperature in dilute solution (in THF). The chain conformations of both polypeptoids are relatively insensitive to temperature in dilute solution.

\subsection{Polymer chain models for SANS data fitting}

SANS data fitting was carried out in the SasView software, using the flexible cylinder model with excluded volume. The chain model is a parametrization of simulations of discrete representation of the wormlike chain model of Kratky and Porod applied in the 
pseudocontinuous limit with original equations developed by Pedersen and Schurtenberger and the corrected formula was used. ${ }^{6-7}$

The total scattering function of a wormlike chain is given by

$$
I_{W L C}\left(q, L_{C}, b, R\right)=\frac{c M}{\rho N_{A v}} \Delta \rho^{2} S_{W L C}\left(q, L_{C}, b\right) P_{C S}(q, R)+I_{i n c}
$$

where $c$ is the concentration, $M$ is the molecular weight of the polymer, $\rho$ is the mass density, $N_{A v}$ is Avogadro's number, $\Delta \rho$ is the scattering length density difference between the polymer and the solvent, $L_{C}$ is the contour length, $b$ is the Kuhn length, $R$ is the radius of the cylinder, $I_{i n c}$ is the incoherent background scattering. The scattering function from the cross section of a rigid rod is given by

$$
P_{C S}(q, R)=\left(\frac{2 J_{1}(q R)}{q R}\right)^{2}
$$

where $J_{1}(x)$ denotes the Bessel function of the first kind. $S_{W L C}\left(q, L_{C}, b\right)$ is the scattering function of single semiflexible chain with excluded volume effects and is given by

$$
\begin{aligned}
S_{W L C}\left(q, L_{C}, b\right) & =\left[1-w\left(q R_{g}\right)\right] S_{\text {Debye }}\left(q, L_{C}, b\right) \\
& +w\left(q R_{g}\right)\left[1.22\left(q R_{g}\right)^{-1 / 0.585}+0.4288\left(q R_{g}\right)^{-2 / 0.585}-1.651\left(q R_{g}\right)^{-3 / 0.585}\right] \\
& +\frac{C\left(n_{b}\right)}{n_{b}}\left\{\frac{4}{15}+\frac{7}{15 u}-\left(\frac{11}{15}+\frac{7}{15 u}\right) \times \exp \left[-u\left(q, L_{C}, b\right)\right]\right\}
\end{aligned}
$$

where

$$
\begin{gathered}
S_{\text {Debye }}\left(q, L_{C}, b\right)=\frac{2}{u\left(q, L_{C}, b\right)}\left\{\exp \left[-u\left(q, L_{C}, b\right)\right]+u\left(q, L_{C}, b\right)-1\right\} \\
u\left(q, L_{C}, b\right)=\frac{L_{C} b}{6}\left\{1-\frac{3}{2 n_{b}}+\frac{3}{2 n_{b}{ }^{2}}-\frac{3}{4 n_{b}{ }^{3}}\left[1-\exp \left(-2 n_{b}\right)\right]\right\} q^{2} \\
n_{b}=\frac{L_{C}}{b} \\
w(x)=\frac{\{1+\tanh [(x-1.523) / 0.1477]\}}{2} \\
\left\langle R_{g}{ }^{2}\right\rangle=\alpha\left(n_{b}\right)^{2} \frac{L_{C} b}{6} \\
u\left(q, L_{C}, b\right)=\alpha\left(n_{b}\right)^{2} q^{2} \frac{L_{C} b}{6}
\end{gathered}
$$




$$
\begin{gathered}
\alpha(x)=\sqrt{\left[1+\left(\frac{x}{3.12}\right)^{2}+\left(\frac{x}{8.67}\right)^{3}\right]^{0.176 / 3}} \\
C\left(n_{b}\right)= \begin{cases}3.06 n_{b}-0.44 & \text { for } L_{C}>10 b \\
1 & \text { for } L_{C} \leq 10 b\end{cases}
\end{gathered}
$$

Fitting parameters include scale, background, contour length $\left(L_{C}\right)$, Kuhn length $(b)$ and radius $(R)$. In particular, the contour lengths were estimated based on the cis/trans ratio of polypeptoid backbone configurations, where an all trans polypeptoid 36-mer will have a contour length of $\sim 13.9 \mathrm{~nm}$, and an all cis polypeptoid 36-mer will have a contour length of $\sim 11 \mathrm{~nm}{ }^{8}$ For the helical polypeptoids, the backbone preferentially adopts cis configuration in the helical geometry with 3 residues per turn and a helical pitch $\sim 6 \AA$, and the overall cis/trans ration is $\sim 3.3 .{ }^{9-11}$ This gives a helix contour length for the helical polypeptoids $\left(L_{C \text {,helix }}\right)$ of $\sim 8.7 \mathrm{~nm}$ and the fitting range for $L_{C \text {,helix }}$ is set to $7.2-10.5 \mathrm{~nm}$. For the coil polypeptoids, a 1:1 cis/trans ratio is estimated as the isomerization energy is relatively low for residues with these types of side chains, ${ }^{12}$ which then gives a chain contour length $\left(L_{C, \text { coil }}\right)$ of $\sim 12.4 \mathrm{~nm}$ and the fitting range for $L_{C, \text { coil }}$ is set to $11-13.9 \mathrm{~nm}$. The fitting range for Kuhn length $(b)$ is set to $1-3 \mathrm{~nm}$. 

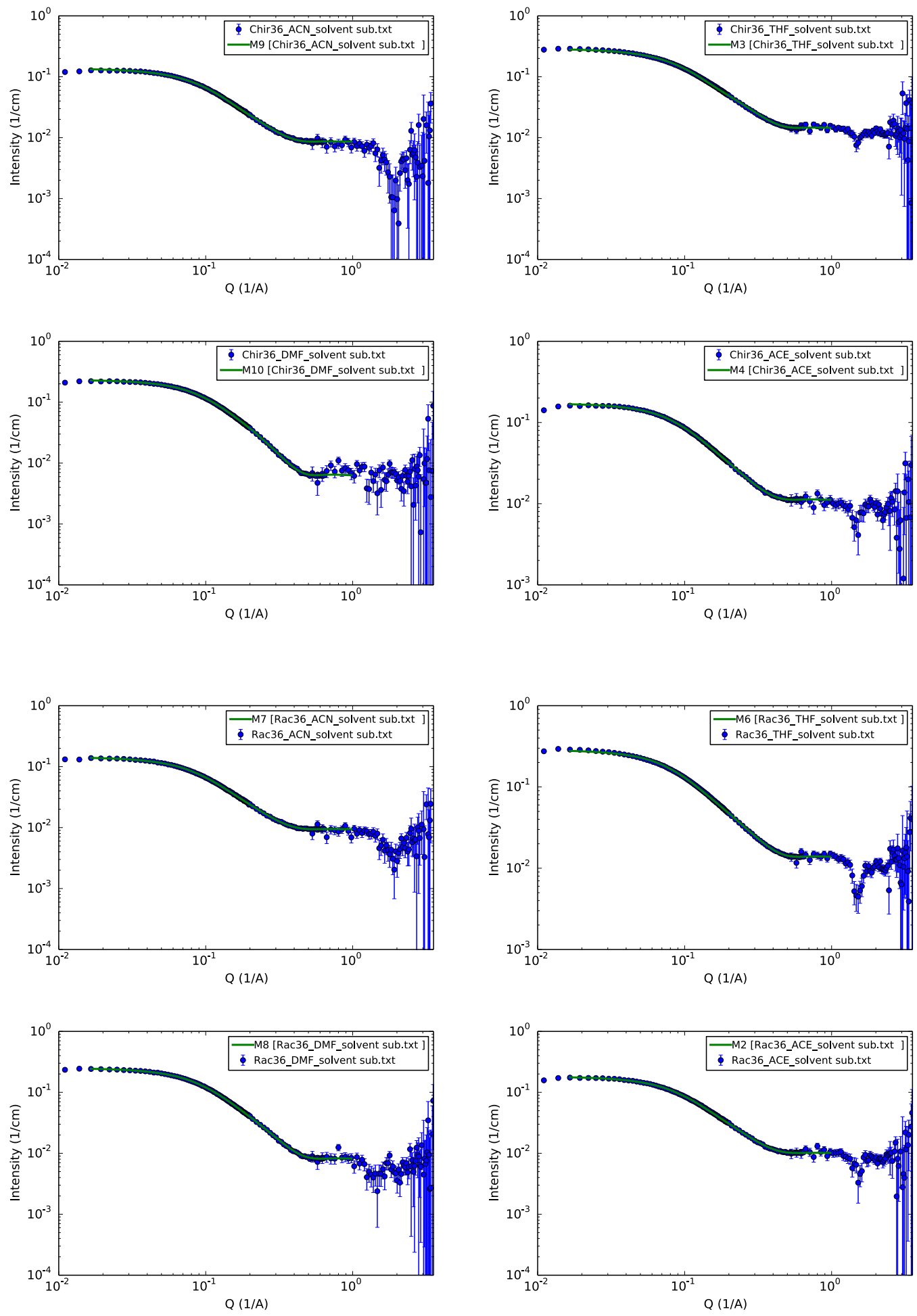

Figure S11. Fitting results of SANS of Chir36 and Rac36 in dilute solutions using the flexible cylinder model with excluded volume in SasView. 
Table S3. Fitting results from the flexible cylinder model with excluded volume in SasView and calculated radius of gyration, $R_{g}$.

\begin{tabular}{ccccccc}
\hline \multirow{4}{*}{ Chir36 } & Solvent & $L(\mathrm{~nm})$ & $b(\mathrm{~nm})$ & $R^{*}(\mathrm{~nm})$ & Chi $^{2}$ & $R_{g}(\mathrm{~nm})$ \\
\hline & ACN & $8.75 \pm 0.24$ & $2.27 \pm 0.12$ & 0.73 & 1.45 & $1.14 \pm 0.04$ \\
& THF & $8.72 \pm 0.08$ & $2.57 \pm 0.05$ & 0.65 & 6.80 & $1.20 \pm 0.02$ \\
& ACE & $8.86 \pm 0.33$ & $1.89 \pm 0.13$ & 0.70 & 1.17 & $1.07 \pm 0.05$ \\
\multirow{3}{*}{ Rac36 } & DMF & $8.68 \pm 0.25$ & $1.79 \pm 0.10$ & 0.69 & 1.19 & $1.04 \pm 0.04$ \\
& ACN & $12.81 \pm 0.59$ & $1.21 \pm 0.09$ & 0.70 & 0.99 & $1.17 \pm 0.06$ \\
& THF & $9.78 \pm 0.18$ & $2.00 \pm 0.07$ & 0.65 & 5.83 & $1.17 \pm 0.03$ \\
& ACE & $12.30 \pm 0.15$ & $1.23 \pm 0.00$ & 0.65 & 1.44 & $1.15 \pm 0.01$ \\
& DMF & $11.80 \pm 0.11$ & $1.18 \pm 0.00$ & 0.64 & 1.22 & $1.10 \pm 0.01$ \\
\hline
\end{tabular}

*Radius $(R)$ has negligible errors from the fitting, therefore errors of $R$ are not listed.

\subsection{Thermal blob calculations}

To see whether the polymer chain conformations are dominated by excluded volume interactions in this case, the thermal blob size $\xi_{T}$ (corresponds to a thermal energy $k_{B} T$ ) is calculated. On length scales smaller than $\xi_{T}$, the excluded volume interactions are weaker than the thermal energy $k_{B} T$, and the conformations of these small sections of the chain are nearly ideal. The calculations are based on scaling approaches in Rubinstein and Colby’s. ${ }^{13}$

The chain interaction parameter, $z$, is related to the second virial coefficient, $A_{2}$ :

$$
Z \approx \frac{A_{2} M^{1 / 2} M_{0}^{3 / 2}}{N_{A v} b^{3}},|z|<1
$$

where $M$ and $M_{0}$ is the molecular weight of the polymer and a Kuhn monomer, respectively, $b$ is the Kuhn length and $N_{A v}$ is Avogadro's number. In solvents near the $\theta$-temperature (i.e., the interaction parameter $|z|<1$ ), the excluded volume $\mathrm{v}$ of a Kuhn monomer has the relation with $A_{2}$ :

$$
\mathrm{v}=\frac{2 M_{0}{ }^{2}}{N_{A v}} A_{2}
$$

The size of the thermal blob

$$
\xi_{T} \approx \frac{b^{4}}{|\mathrm{v}|}
$$


and the number of monomers in a thermal blob

$$
g_{T} \approx \frac{b^{6}}{\mathrm{v}^{2}}
$$

Based on the calculation (Table S3), the thermal blob size $\left(\xi_{T}\right)$ is always around or larger than the chain size $\left(R_{g}\right)$. Therefore, for these polypeptoids in dilution solution examined here, the excluded volume interactions have minimal effects in terms of swelling the chains (the fact that $z$ $\lesssim 1$ also indicates that the excluded volume interactions are weak).

Table S4. Thermal blob calculations

\begin{tabular}{ccccccc}
\hline \multirow{4}{*}{ Chir36 } & Solvent & $A_{2} * 10^{3}\left(\mathrm{~cm}^{3} \mathrm{~mol} \mathrm{~g}^{-2}\right)$ & $z$ & $\mathrm{v}\left(\mathrm{nm}^{3}\right)$ & $\xi_{T}(\mathrm{~nm})$ & $g_{T}$ \\
\hline \multirow{6}{*}{ Rac36 } & ACN & 0.288 & 0.142 & 1.69 & 15.68 & 47.7 \\
& THF & 0.385 & 0.159 & 2.92 & 14.93 & 33.8 \\
& ACE & 0.545 & 0.347 & 2.17 & 5.89 & 9.7 \\
& DMF & 1.833 & 1.307 & 6.81 & 1.51 & 0.7 \\
\hline \multirow{3}{*}{} & ACN & 0.195 & 0.140 & 0.15 & 14.10 & 135.8 \\
& THF & 0.431 & 0.218 & 1.57 & 10.16 & 25.8 \\
& ACE & 0.650 & 0.483 & 0.57 & 4.03 & 10.7 \\
& DMF & 1.455 & 1.223 & 1.27 & 1.53 & 1.7 \\
\hline
\end{tabular}

\section{References}

1. Yu, B.; Danielsen, S. P. O.; Patterson, A. L.; Davidson, E. C.; Segalman, R. A. Effects of Helical Chain Shape on Lamellae-Forming Block Copolymer Self-Assembly. Macromolecules 2019, 52 (6), 2560-2568.

2. Arnold, O.; Bilheux, J. C.; Borreguero, J. M.; Buts, A.; Campbell, S. I.; Chapon, L.; Doucet, M.; Draper, N.; Leal, R. F.; Gigg, M. A.; Lynch, V. E.; Markvardsen, A.; Mikkelson, D. J.; Mikkelson, R. L.; Miller, R.; Palmen, K.; Parker, P.; Passos, G.; Perring, T. G.; Peterson, P. F.; Ren, S.; Reuter, M. A.; Savici, A. T.; Taylor, J. W.; Taylor, R. J.; Tolchenoy, R.; Zhou, W.; Zikoysky, J. Mantid-Data analysis and visualization package for neutron scattering and mu SR experiments. Nucl. Instrum. Meth. A 2014, 764, 156-166.

3. Wignall, G. D.; Bates, F. S. Absolute Calibration of Small-Angle Neutron-Scattering Data. J. Appl. Crystallogr. 1987, 20, 28-40. 
4. Theisen, A.; Johann, C.; Deacon, M. P.; Harding, S. E. Refractive Increment Data-Book for Polymer and Biomolecular Scientists. Nottingham University Press: Nottingham, United Kingdom, 2000.

5. Huglin, M. B. Specific Refractive Index Increments of Polymer Solutions. I. Literature Values. J. Appl. Polym. Sci. 1965, 9 (12), 3963-4001.

6. Pedersen, J. S.; Schurtenberger, P. Scattering Functions of Semiflexible Polymers with and without Excluded Volume Effects. Macromolecules 1996, 29 (23), 7602-7612.

7. Chen, W.-R.; Butler, P. D.; Magid, L. J. Incorporating Intermicellar Interactions in the Fitting of SANS Data from Cationic Wormlike Micelles. Langmuir 2006, 22 (15), 6539-6548.

8. Hudson, B. C.; Battigelli, A.; Connolly, M. D.; Edison, J.; Spencer, R. K.; Whitelam, S.; Zuckermann, R. N.; Paravastu, A. K. Evidence for cis Amide Bonds in Peptoid Nanosheets. $J$. Phys. Chem. Lett. 2018, 9 (10), 2574-2578.

9. Wu, C. W.; Sanborn, T. J.; Huang, K.; Zuckermann, R. N.; Barron, A. E. Peptoid Oligomers with $\alpha$-Chiral, Aromatic Side Chains: Sequence Requirements for the Formation of Stable Peptoid Helices. J. Am. Chem. Soc. 2001, 123 (28), 6778-6784.

10. Armand, P.; Kirshenbaum, K.; Falicov, A.; Dunbrack, R. L.; Dill, K. A.; Zuckermann, R. N.; Cohen, F. E. Chiral N-substituted glycines can form stable helical conformations. Folding Des. 1997, 2 (6), 369-375.

11. Stringer, J. R.; Crapster, J. A.; Guzei, I. A.; Blackwell, H. E. Extraordinarily robust polyproline type I peptoid helices generated via the incorporation of alpha-chiral aromatic N-1naphthylethyl side chains. J. Am. Chem. Soc. 2011, 133 (39), 15559-67.

12. Shah, N. H.; Butterfoss, G. L.; Nguyen, K.; Yoo, B.; Bonneau, R.; Rabenstein, D. L.; Kirshenbaum, K. Oligo(N-aryl glycines): A New Twist on Structured Peptoids. J. Am. Chem. Soc. 2008, 130 (49), 16622-16632.

13. Rubinstein, M.; Colby, R. H. Polymer Physics. Oxford University Press: New York, 2003. 